\title{
The Behavior of Weighted Graph's Orbit and Its Energy
}

\author{
Ali A. Shukur $\mathbb{D}^{1,2}$ Akbar Jahanbani $\mathbb{D}^{3}$, and Haider Shelash $^{4}$ \\ ${ }^{1}$ Computer Technical Engineering Department, College of Technical Engineering, The Islamic University, Najaf, Iraq \\ ${ }^{2}$ Mechanics-Mathematics Faculty, Belarusian State University, Minsk, Belarus \\ ${ }^{3}$ Department of Mathematics, Azarbaijan Shahid Madani University, Tabriz, Iran \\ ${ }^{4}$ Department of Mathematics, University of Kufa, Najaf, Iraq
}

Correspondence should be addressed to Akbar Jahanbani; akbar.jahanbani92@gmail.com

Received 29 March 2021; Revised 17 April 2021; Accepted 5 May 2021; Published 17 May 2021

Academic Editor: Andrea Semaničová-Feňovčíková

Copyright ( 2021 Ali A. Shukur et al. This is an open access article distributed under the Creative Commons Attribution License, which permits unrestricted use, distribution, and reproduction in any medium, provided the original work is properly cited.

Studying the orbit of an element in a discrete dynamical system is one of the most important areas in pure and applied mathematics. It is well known that each graph contains a finite (or infinite) number of elements. In this work, we introduce a new analytical phenomenon to the weighted graphs by studying the orbit of their elements. Studying the weighted graph's orbit allows us to have a better understanding to the behaviour of the systems (graphs) during determined time and environment. Moreover, the energy of the graph's orbit is given.

\section{Introduction}

Let $G$ be a graph of order $N, E(G)=\left\{u_{1}, u_{2}, \ldots, u_{N}\right\}$ be the set of edges, and $V(G)=\left\{v_{1}, v_{2}, \ldots, v_{N}\right\}$ be the set of vertices. We consider connected graphs with weights. An (edge)-weighted graph $W(G)$ is defined to be an ordered pair $(G, f)$, where $G_{N}$ is the underlying graph of order $N$ and

$$
f: E \longrightarrow \mathbb{R}
$$

is the weight function, which assigns to each edge $u \in E(G)$ a nonzero weight $f(u)$. Every graph can be regarded as the weighted graph with weight of each edge equal to one. Thus, weighted graphs are generalizations of graphs.

In this paper, we study the orbit of weighted graph for given initial weight-edge $u$ : $=u_{1}=u_{2}=\cdots=u_{N}$ such as

$$
\operatorname{Orb}\left(G_{N}, f(u)\right)=\left\{u, f(u), f^{2}(u), \ldots, f^{n}(u): n \in \mathbb{N}, u \in E(G)\right\}
$$

and by $G^{n}(W(G), f(u))$ we denote the system graph's orbit of order $N$ but in short, we will use only $G^{n}$. Usually, the algebraic and topological peculiarities of graphs bring information about it in present time, but studying graph's orbit shows how does graph behave during the time. For more details about discrete dynamical systems, we refer $[1,2]$. 


\section{Linear and Chaotic Behavior of Weighted Graphs}

In this section, we discuss properties of the weighted graphs necessary for other discussions.

We recall basic definitions.

A metric space defined over a set of points in terms of distances in a graph defined over the $V(G)$ is called a graph metric. The vertex set (of an undirected graph) and the distance function form a metric space, if and only if the graph is connected.

Let us assume that the function in (1) represents the distance between $v_{j}$ and $v_{j}$ where $i \neq j$ such as $f(u)=\max \left|\operatorname{dist}\left(v_{i}, v_{j}\right)\right|$, i.e., metric edge map over metric space. One can easily check the following statement.

Proposition 1. Let $G_{N}$ be a connected weighted graph of $\operatorname{order} N$ and $f(u)$ be a metric edge map. Then,

Case I. If $f(u)$ is linear decreasing map, then the graph's orbit behaves like

$$
G^{n} \longrightarrow 0 \text { as } n \longrightarrow \infty
$$

In this case, we say that the graph is attracted and denote it by $G_{A}^{n}$.

Case II. If $f(u)$ is linear increasing map, then the graph's orbit behaves like

$$
G^{n} \longrightarrow \infty \text { as } n \longrightarrow \infty \text {. }
$$

In this case, we say that the graph is repelled and denote it by $G_{R}^{n}$.

Example 1. Let $G \cong K_{3}$ and $f(u)=(1 / 2) u$ with initial edges $u:=u_{1}=u_{2}=u_{3}=(1 / 2)$. Then, the orbit of $\left(K_{3}\right)^{n}$ is as follows:

$$
\operatorname{Orb}\left(\left(K_{3}\right)^{n}, \frac{1}{2} u\right)=\left\{\frac{1}{2}, \frac{1}{4}, \frac{1}{16}, \frac{1}{64}, \frac{1}{256}, \frac{1}{1024}, n=0,1,2, \ldots, 5\right\},
$$

and the attracted graph's orbit $\left(K_{3}\right)^{n}$ for $n=1,2, \ldots, 5$ is shown in Figure 1.

Smilar to the above considered techniques, we will check the orbit of $K_{3}$ where $f(u)=2 u$ with initial edges:

$$
\operatorname{Orb}\left(\left(K_{3}\right)^{n}, 2 u\right)=\{2,4,16,64,256,1024 ; n=0,1,2, \ldots, 5\},
$$

and the repelled graph's orbit of $\left(K_{3}\right)^{n}$ for $n=1,2, \ldots, 5$ is shown in Figure 2.

In what follows, we will consider a special case when function (1) is a nonlinear chaotic map; here, the graph's orbit $G^{n}$ shows unpredictable behavior. We say that the graph's orbit has chaotic index.

Since Lorenz discovered his nonlinear system in 1964 [3], chaos had been studied as a system with conditions. After that, chaotic maps find their way in many scientific branches.

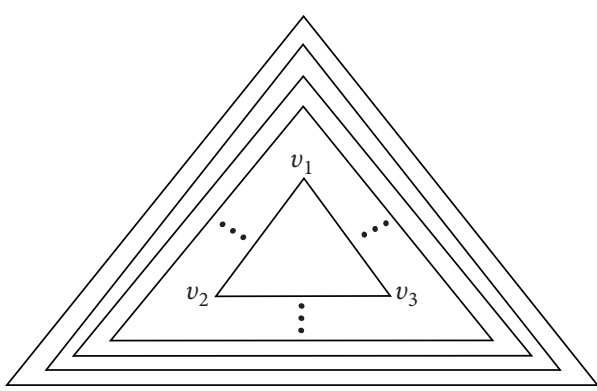

Figure 1: Attracted graph's orbit of $K_{3}$.

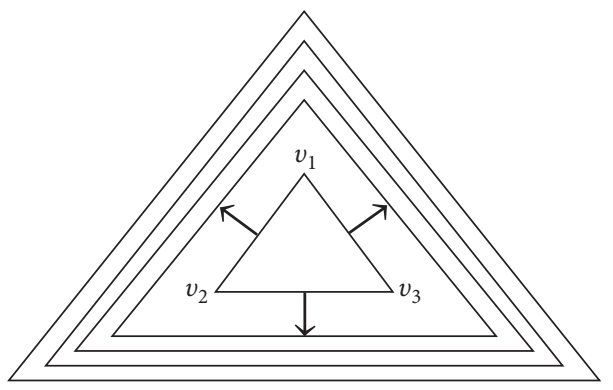

FIGURE 2: Repelled graph's orbit of $K_{3}$.

Among the chaotic maps, a logistic map is the most studied one.

The logistic map is a polynomial mapping, and it was popularized in 1976 [4] by the biologist Robert May as an analogous discrete-time demographic model. Nowadays, the logistic map is considered as the simplest nonlinear dynamical system in dimension one which is given by the following expression:

$$
x_{n+1}=\mu x_{n}\left(1-x_{n}\right),
$$

for $x \in(0,1)$ and $\mu \in[0,4]$. The logistic map shows a very special behavior which is complex and chaotic as one can see in Figure 3.

Coming back to our issue, we will assume that the function in (1) is logistic map such that $u_{n+1}=f(u)$ and

$$
u_{n+1}=\mu u_{n}\left(1-u_{n}\right) \text {. }
$$

To better understand, we give the following examples.

Example 2. Let the parameter of (8) $\mu=3.56995$. In Table 1 and Figure 4, we observed that the graph's orbit of $\left(K_{3}\right)^{n}$ for $n=1,2, \ldots, 7$ with initial edges $u:=u_{1}=u_{2}=u_{3}=0.25$ shows a nonlinear behaviour under the action of logistic map settled with the mentioned $\mu$.

Example 3. The Cayley's orbit graph Cay $\left(D_{16}, S\right)$ of the Dihedral group of order 16 when

$$
S=\left\{a, a^{7}, b\right\}
$$

generated by logistic map considered in Example 2, is shown in Figure 5. 


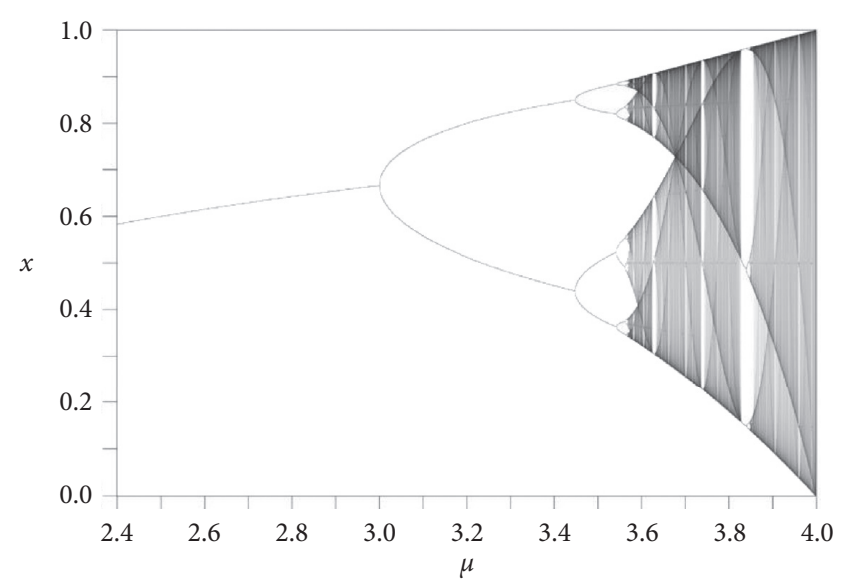

FIgURE 3: The bifurcation diagram of logistic map for $x \in(0,1)$ and $\mu \in[0,4]$.

TABLE 1: The values of graph's orbit of $K_{3}$ under the action of logistic map.

\begin{tabular}{ll}
\hline$x_{0}=0.24$ & $x_{1} \approx 0.6$ \\
$x_{2} \approx 0.8$ & $x_{3} \approx 0.5$ \\
$x_{4} \approx 0.8$ & $x_{5} \approx 0.5$ \\
$x_{6} \approx 0.8$ & $x_{7} \approx 0.5$ \\
\hline
\end{tabular}

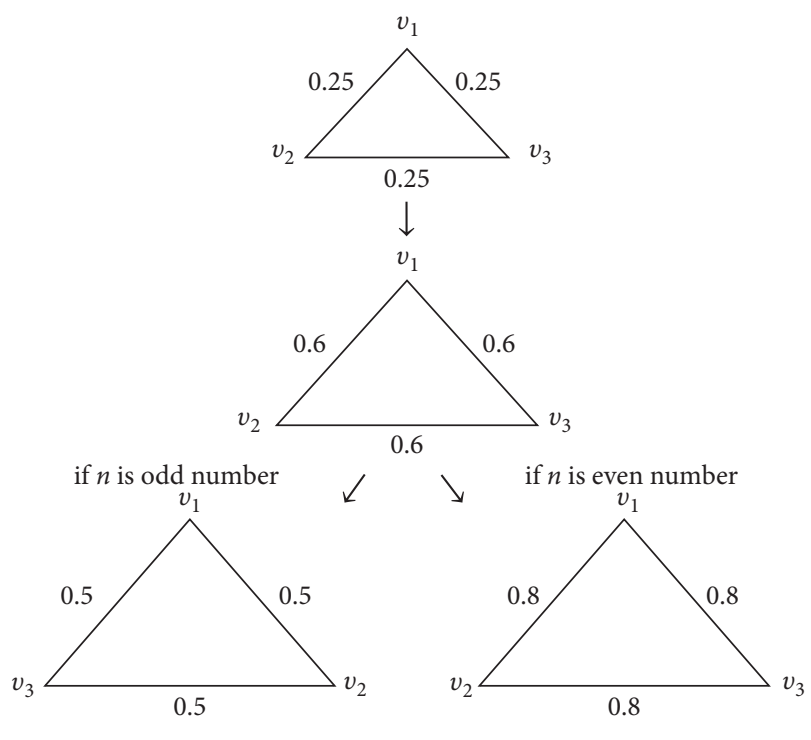

Figure 4: Chaotic graph's orbit of $K_{3}$.

\section{Energy of Weighted Graph's Orbit}

In this section, we are interested in studying one of the most important topological graph indices for $G^{n}$ which was termed by graph energy.

Let $W(G)$ be a graph of order $N$, and the adjacency matrix of a weighted graph $W(G)$ is the $N \times N$ matrix $A(W(G))=\left(w_{i, j}\right)$, where

$$
w_{i j}= \begin{cases}w\left(v_{i}, v_{j}\right), & \text { if } v_{i} v_{j} \in E(W(G)), \\ 0, & \text { otherwise. }\end{cases}
$$

The matrix $A(W(G))$ is real symmetric, so all its eigenvalues are real. The characteristic polynomial $\Phi(W(G), \lambda)=|\lambda I-A(W(G))|$ of the matrix $A(W(G))$ is called the characteristic polynomial of the weighted graph $W(G)$. The eigenvalues of $A(W(G))$ are called the eigenvalues of $W(G)$. The set of distinct eigenvalues of $W(G)$ together with their multiplicities is called the spectrum of $W(G)$. The energy of weighted graph was defined in [5] as follows:

$$
E(W(G))=\sum_{i=1}^{N}\left|\lambda_{i}\right|
$$

where $\lambda_{1}, \lambda_{2}, \ldots, \lambda_{N}$ are the eigenvalues of $A(W(G))$.

This graph invariant in (11) has important applications in chemical graph theory and has been extensively studied. Moreover, in chemical graph theory, if the underlying molecule is a hydrocarbon, then $G$ is a simple, unweighted graph but if the conjugated molecule contains atoms different from carbon and hydrogen (in chemistry referred to as "heteroatoms"), then $G$ must possess pertinently weighted edges (see [6]). These weights are usually positive valued, but they may also be negative (for more details, we refer to [7-12]).

Let us denote by $\mathbb{M}\left(A\left(G^{n}\right)\right)$ the set of the adjacency matrices of graph's orbit $G^{n}$ for $n \in \mathbb{N}$ such that the elements of each adjacency matrix depends on the order of considered map and its nature (linear or nonlinear). Therefore, the energy of $G^{n}$ is the sum of the energies which is calculated by the adjacency matrices in graph's orbit that can be given by the following formula:

$$
\sum_{l=0}^{n}\left[\sum_{i=0}^{N}\left|\lambda_{i}\right|\right]_{l}
$$

In particular, one can see that we have 5 different values in graph's orbit of $\left(K_{3}\right)^{n}$ considered in Example 1; this means that we almost have 5 adjacency matrices for each one and the energy of graph's orbit is the sum of all energies calculated for each adjacency matrix belonging to the calculated orbit. Thus, we have

$$
E\left(K_{3, A}^{n}\right) \approx 2+1+0.25+0.0624+0.0156+0.004=3.332,
$$

while the energy in case II is

$$
E\left(K_{3, R}^{n}\right) \approx 8+16+64+256+1024+4096=5464 .
$$

It is obvious that

$$
E\left(G_{A}^{n}\right)<E\left(G_{R}^{n}\right)
$$

More precisely, if map (1) is logistic map given by (8) where $\mu=3.56995$ with initial edge $u \in[0,1]$, it follows that the graph's orbit $G^{n}$ is chaotic and unpredictable. To have more, we assume that the graph's orbit $G^{n}$ contains the following adjacency matrices:

(1) $A_{1}(G(W))$ is the adjacency matrix of the initial weight edges of weighted graph $G(W)$. 


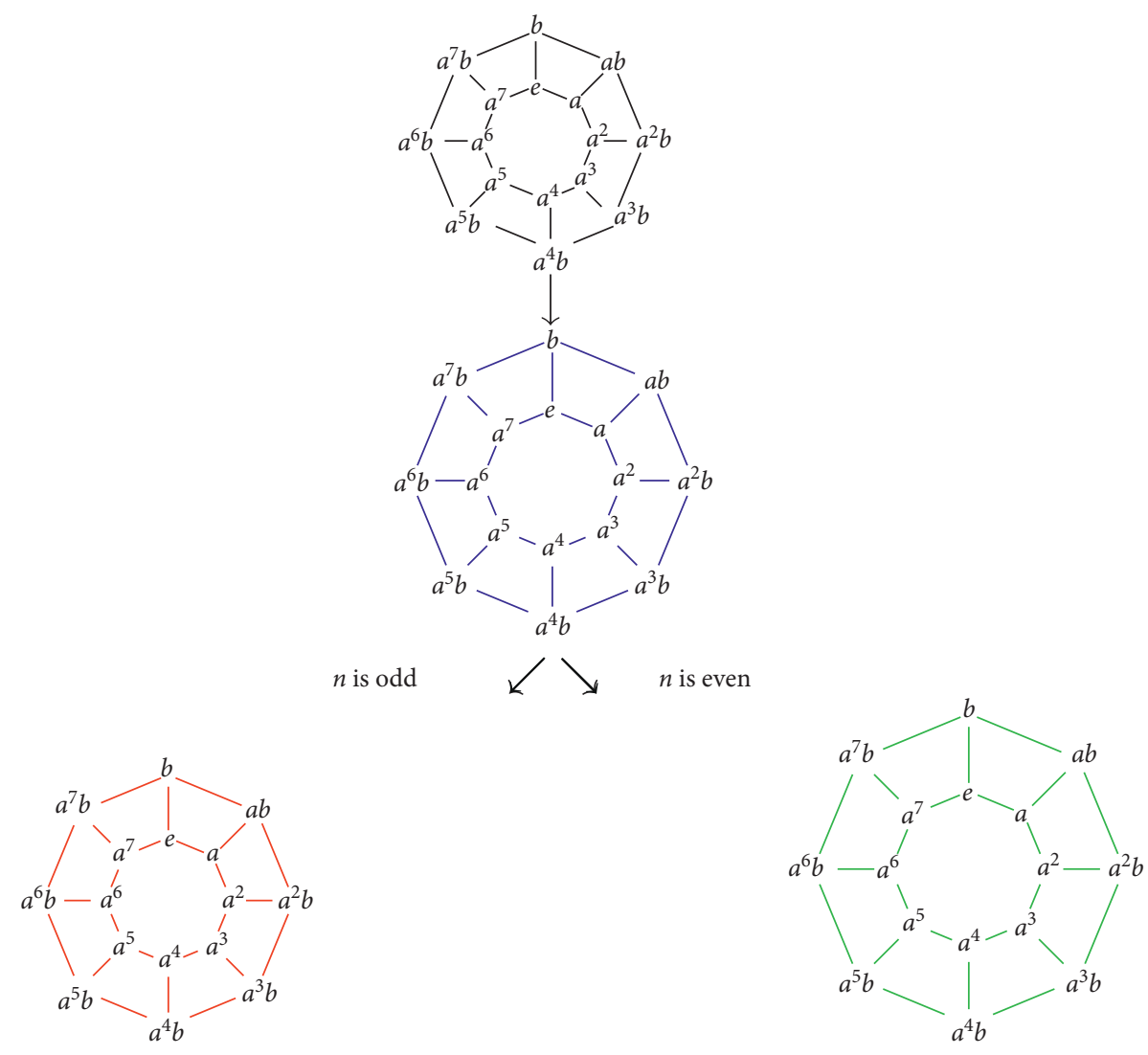

Figure 5: Graph's orbit of Cay $\left(D_{1} 6, S\right)$ in which the black color represents the graph status at the initial edge, the blue color represents the graph status at the first order, the red color shows the graph status for odd orders, and green color shows the graph status for even orders.

(2) $A_{2}(G(W))$ is the adjacency matrix of weighted graph $G(W)$ obtained by the action of $f(u)$.

(3) $A_{\alpha}(G(W))$ is the adjacency matrix of weighted graph $\mathrm{G}(\mathrm{W})$ obtained by the action of $f^{2 n}(u)(n \in \mathbb{N})$.

(4) $A_{\beta}(G(W))$ is the adjacency matrix of weighted graph $G(W)$ obtained by the action of $f^{2 n+1}(u)(n \in \mathbb{N})$.

Hence, we can calculate the energy of graph $G^{n}$ as follows:

(1) If $n$ is even, then

$$
E\left(G^{n}\right)=\left[\sum_{i=1}^{N}\left|\lambda_{i_{1}}\right|+\sum_{i=1}^{N}\left|\lambda_{i_{2}}\right|+\left(\frac{n}{2}\right) \sum_{i=1}^{N}\left|\lambda_{i_{\alpha}}\right|+\left(\frac{n}{2}-1\right) \sum_{i=1}^{N}\left|\lambda_{i_{\beta}}\right|\right] .
$$

(2) If $n$ is odd, then

$$
E\left(G^{n}\right)=\left[\sum_{i=1}^{N}\left|\lambda_{i_{1}}\right|+\sum_{i=1}^{N}\left|\lambda_{i_{2}}\right|+\left(\frac{n-1}{2}\right) \sum_{i=1}^{N}\left|\lambda_{i_{\alpha}}\right|+\left(\frac{n-1}{2}\right) \sum_{i=1}^{N}\left|\lambda_{i_{\beta}}\right|\right],
$$

where $\lambda_{i_{1}}, \lambda_{i_{2}}, \lambda_{i_{\alpha}}$, and $\lambda_{i_{\beta}}$ are the eigenvalues of $A_{1}, A_{2}, A_{\alpha}$, and $A_{\beta}$, respectively. In particular, the energy of $\left(K_{3}\right)^{n}$ considered in Example 2 is

$$
E\left(\left(K_{3}\right)^{n}\right) \approx 1+2.4+(3) 3.2+(3) 2=19
$$

Moreover, by using formula (19), one can apply the earlier results of weighted graph energy to their orbit's graph, i.e., to the set $\mathbb{M}\left(A\left(G^{n}\right)\right)$. Here, we are considering a special class called bipartite weighted graphs for which the characteristic polynomials are determined in the following.

Lemma 1. Let $G$ be a bipartite weighted graph with $n$ vertices. Then,

$$
\Phi(G, q)=\sum_{0}^{n / 2} b(G, k) q^{2 k}(-1)^{k} q^{n-2 k}
$$

where $b(G, k) \geq 0$ for all $k$.

Equation (19) is widely used in the theory of graph energy for unweighted graphs and weighted bipartite graphs. In [13], it was shown that if the graph $G$ is a bipartite graph with the characteristic polynomial as in (19), then from the Coulson integral formula follows the energy of $G$ :

$$
E(G)=\frac{2}{\pi} \int_{0}^{\infty} \frac{1}{q^{2}} \ln \left(\sum_{0}^{n / 2} b(G, k) q^{2 k}\right) \mathrm{d} q .
$$

The energy in (20) holds equally for simple and bipartite weighted graph. Moreover, the energy of weighted graphs orbit can be calculated by using (20). In [14], it was shown 
TABLE 2: Energy of some chemical systems represented by weighted graphs in Figure 6 .

\begin{tabular}{|c|c|c|c|c|c|}
\hline Object & $u$ & $N$ & $n$ & $E\left(G^{n}\right)$ & Graph \\
\hline \multirow{3}{*}{ Vinlye-chloride-like systems } & \multirow{3}{*}{0.4} & \multirow{3}{*}{3} & 2 & 6 & \multirow{3}{*}{$\Gamma_{1}$} \\
\hline & & & 5 & 12.2 & \\
\hline & & & 9 & 20.4 & \\
\hline \multirow{3}{*}{ Pyrrole-like systems } & \multirow{3}{*}{0.2} & \multirow{3}{*}{5} & 2 & 19.6 & \multirow{3}{*}{$\Gamma_{2}$} \\
\hline & & & 4 & 30.8 & \\
\hline & & & 7 & 50.4 & \\
\hline \multirow{3}{*}{ Pyridine-like systems } & \multirow{3}{*}{0.9} & \multirow{3}{*}{6} & 2 & 32.7 & \multirow{3}{*}{$\Gamma_{3}$} \\
\hline & & & 5 & 47.4 & \\
\hline & & & 8 & 71.1 & \\
\hline \multirow{3}{*}{ 1,1-Dichloro-ethylene-like systems } & \multirow{3}{*}{0.4} & \multirow{3}{*}{4} & 2 & 10.8 & \multirow{3}{*}{$\Gamma_{4}$} \\
\hline & & & 5 & 20.2 & \\
\hline & & & 8 & 30.9 & \\
\hline
\end{tabular}
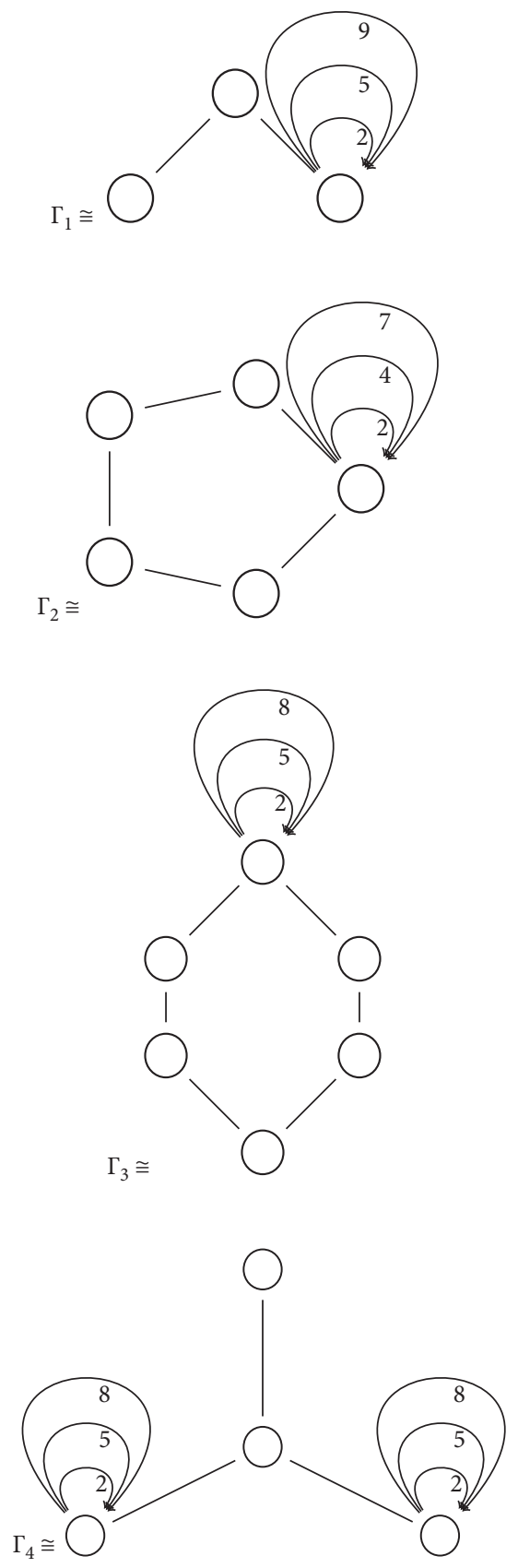

Figure 6: The graphs $\Gamma_{i}, i=1,2,3,4$. 
that the weighted star $W\left(K_{1, N-1}\right)$ on $N$ vertices with $w_{i j}$ on the edge $e_{k}=\left(v_{i}, v_{j}\right)$, where $1 \leq i, j \leq N$ and $1 \leq k \leq N-1$, can be obtained by the following.

$$
E\left(W\left(K_{1, N-1}\right)\right)=2 \sqrt{w_{12}^{2}+w_{13}^{2}+\cdots+w_{1 N}^{2}} .
$$

Proposition 2. The energy of weighted star graph orbit is

$$
E\left(\left(W\left(K_{1, N-1}\right)\right)^{n}\right)=2 n \sum_{l=0}^{n}\left[\sqrt{\left.\sum_{r=1}^{N} w_{1 r}^{2}\right]_{l} .}\right.
$$

The proof is direct from (19) and (21).

\section{Computational Studies on Weighted Graph Orbit}

Studying the dynamics of such physical element (molecular) allows us to understand the behavior of it during determined time and environment. To have a better insight into the particularities of the weighted graph orbit, we investigate the orbit of some chemical systems (as mentioned above, the heteroatom systems can be represented by graphs with weights) under the action of the logistic map given in (8) with different times as shown in Table 2.

\section{Conclusion}

The weighted graphs are very important in representing many problems in complex networks, data structure, chemical systems, urban engineering, and others. In Proposition 1, we show that the introduced method of studying the orbit of each weighted edge belongs to weighted graph brings us more information about the behavior of the consider dynamical systems. We show the relationship between the weight function and the graph's orbit with three possible cases and compute some examples for better understanding their properties. By computing the energy of graph's orbit, we show the change of the energy of such a system during the time which was given in Proposition 2. Finally, we computed the energy of graph's orbit of some popular chemical systems.

\section{Data Availability}

The data used to support the findings of this study are included within the article.

\section{Conflicts of Interest}

The authors declare that they have no conflicts of interest.

\section{References}

[1] H. S. Mortveit, An Introduction to Sequential Dynamical Systems, Springer, NewYork, NY, USA, 2008.

[2] A. B. Antonevich and A. A. Shukur, "On powers of operator generated by rotation," Journal of Analysis and Applications, vol. 16, pp. 57-67, 2018.
[3] E. N. Lorenz, "Deterministic nonperiodic flow," Journal of the Atmospheric Sciences, vol. 20, no. 2, pp. 130-141, 1963.

[4] R. M. May, "Simple mathematical models with very complicated dynamics," Nature, vol. 261, no. 5560, pp. 459-467, 1976.

[5] I. Gutman and J.-Y. Shao, "The energy change of weighted graphs," Linear Algebra and Its Applications, vol. 435, no. 10, pp. 2425-2431, 2011.

[6] R. B. Mallion, A. J. Schwenk, and N. Trinajstich, "A graphical study of heteroconjugated molecules," Croatica Chemica Acta, vol. 46, pp. 171-182, 1974.

[7] E. Andrade, M. Robbiano, and B. San Martín, "A lower bound for the energy of symmetric matrices and graphs," Linear Algebra and Its Applications, vol. 513, pp. 264-275, 2017.

[8] D. Cvetkovic, M. Doob, and H. Sachs, Spectra of GraphsTheory and Application, Academic Press, Cambridge, MA, USA, 1980.

[9] I. Gutman, "The energy of a graph," Ber Math-Statist Sekt Forschungsz Graz, vol. 103, pp. 1-22, 1978.

[10] I. Gutman, "Comparative studies of graph energies," Bulletin Classe de Sciences Mathematiques et Naturelles, Sciences Mathematiques, vol. 144, pp. 1-17, 2012.

[11] I. Gutman, "Census of graph energies," MATCH Communications in Mathematical and in Computer Chemistry, vol. 74, pp. 219-221, 2015.

[12] S. Ali and I. Gutman, "Energy of monad graphs," Bulletin of the International Mathematical Virtual Institute, vol. 11, no. 2, pp. 261-268, 2021.

[13] I. Gutman, "Acyclic systems with extremal Hückel $\pi$-electron energy," Theoretica Chimica Acta, vol. 45, no. 2, pp. 79-87, 1977.

[14] H. A. Ganie and B. A. Chat, "Bounds for the energy of weighted graphs," Discrete Applied Mathematics, vol. 268, pp. 91-101, 2019. 